\title{
Expiratory muscle activity in preterm babies
}

\author{
M SOUTH, C J MORLEY, AND G HUGHES \\ Department of Paediatrics, University of Cambridge, Addenbrooke's Hospital, Cambridge
}

SUMMARY The activity of the right external abdominal oblique muscle, in relation to respiration, in newborn babies from 25 to 34 weeks' gestation was studied, using surface electrodes. Babies without respiratory difficulties showed no activity associated with respiration in these muscles. Grunting babies with respiratory disease showed pronounced activity during expiration. This activity continued when the babies were endotracheally intubated; if the babies were ventilated it sometimes disturbed the tidal ventilation delivered by the machines. When the baby expired during a ventilator inflation, so that gas came up the endotracheal tube against the inflating pressure, the abdominal oblique muscles contracted in $98 \%$ of the breaths.

In adults certain muscles of the abdominal wall are active during forced expiratory manoeuvres. ${ }^{\prime}$ What knowledge exists on the activity of these muscles in preterm babies suggests that they may be active during expiration in the presence of respiratory disease. ${ }^{2}$ Previous work from our department has shown that most babies on ventilators make spontaneous respiratory efforts. ${ }^{3}$ When a ventilated baby breathes out during a ventilator inflation and disturbs the tidal volume delivered by that inflation, it increases the risk of pneumothorax. ${ }^{+}$This pattern of interaction between the baby and the ventilator was termed 'active expiration', because it was thought that expiratory muscle activity played a part. ${ }^{4}$ This suggestion has been questioned. ${ }^{5}$

To investigate the activity of the expiratory muscles we developed a technique of abdominal muscle electromyography, which we have used to study newborn babies in an attempt to answer the following questions: do preterm babies have expiratory muscle activity, and if they do, under what circumstances does it occur?

We chose to study the external abdominal oblique muscle. The fibres arise from the lower eight ribs, passing anteriorly and caudally to the anterior half of the iliac crest and a fibrous aponeurosis which continues to the midline. Contraction of the muscles compresses the abdominal contents and pushes up the diaphragm; it also draws the lower ribs downwards and medially. These muscles have been shown to contract during active expiratory efforts in adults but little is known of their activity in the preterm baby. ${ }^{\prime}$

\section{Methods}

Three groups of babies were studied during the first few days of life: six well preterm babies ( 28 to 32 weeks' gestation, mean 30 weeks) with no respiratory illness; five babies with the respiratory distress syndrome who were grunting, and 34 babies with respiratory distress syndrome who were intubated and ventilated ( 25 to 34 weeks' gestation, mean 29 weeks). The babies on ventilators were selected at random from the intensive care nursery over six months. They were studied once during the first week of life. Only babies who could be seen to be making respiratory efforts while being ventilated were studied. Babies receiving neuromuscular blocking agents were not selected; nor were those termed clinically 'unstable'.

The babies were studied in their cots or incubators, in the supine position, and at a time when they were quiet, making no gross body movements. No further assessment of arousal state was made. All, apart from the six well babies, were studied in the intensive care section of the neonatal unit, where there are many sources of electrical interference.

Full details of the technique for recording the electromyogram (EMG) potentials from the external abdominal oblique muscle are reported elsewhere. ${ }^{6}$ We used standard self-adhesive neonatal electrocardiogram electrodes placed over the right external abdominal oblique muscle, parallel to the direction of the muscle fibres and along a line perpendicular to the electrocardiogram vector at this point. This results in the best EMG signal with 
least interference from the electrocardiogram. Signal filtering was kept to the minimum consistent with obtaining interpretable signals, and consisted of $50 \mathrm{~Hz}$ notch filtering, a low cut off filter (set between 0.3 and $1.0 \mathrm{~Hz}$ ) to reduce movement artefacts, and a high cut off filter (set between 300 and $1000 \mathrm{~Hz}$ ) to reduce interference from electrical apparatus (particularly electrocardiogram monitors). The EMG signals were compared with the babies respiratory cycles, which were recorded from a pneumotachograph and an abdominal respiration monitor.

The well babies and those who were grunting with respiratory distress syndrome had masks that incorporated the pneumotachograph, which was placed gently over their faces and from which gas flow and tidal volume could be recorded. A respiration monitor (Graseby-Dynamics MR10) was placed on the abdomen. The signals were recorded on a polygraph. Recordings of the EMG signal were also made without the face mask to determine whether its presence influenced external abdominal oblique muscle activity. If no muscle activity was seen on the EMG signal then a loud handclap was used to stimulate movement in the baby; activity on the EMG trace then confirmed that the apparatus was responding correctly. The six well babies were each studied for at least 30 minutes and the grunting babies for at least 10 minutes. If the grunting babies had been receiving oxygen this was continued during the study.

The thirty four babies with respiratory distress syndrome who were intubated and ventilated were studied in the same way as the other two groups, except that the pneumotachograph was placed in series with the endotracheal tube. Two recordings were performed on each of these babies: firstly a 30 second period during which the baby breathed spontaneously with continuous positive airway pressure (CPAP), and then a period of about 100 ventilator cycles while the baby was receiving intermittent positive pressure ventilation (IPPV). The ventilator settings were those selected by the clinical team, and the level of CPAP used for the period of spontaneous breathing was the same as the end pressure during IPPV. A period without the pneumotachograph in place was also observed to determine if its presence might influence the activity of the external abdominal oblique muscle. The respiration monitor used on the upper abdomens of these babies showed spontaneous respiratory activity with little or no interference from mechanical ventilation. $^{7}$

In each case the calibration of zero flow and volume detection by the monitoring system (pneumotachograph, differential pressure trans- ducer, and electronic integrator) was checked immediately before and after the observation period. In no case had there been any drift in the baselines.

\section{Results}

Comparison of the traces with and without the pneumotachograph or the face mask in place showed no differences in activity of the external abdominal oblique muscle that could be attributed to the presence of these devices.

The well babies. During observation, there was no activity of the external abdominal oblique muscles related to respiration. Activity in these muscles during trunk movements showed that this was not a failure of the recording technique (Fig. 1). Some babies responded to the siting or removal of the face mask by crying, and there was clear evidence of external abdominal oblique muscle activity during the expiratory cry, but not during the intake of breath between cries.

The grunting babies. When audibly grunting, all babies showed noticeable activity in the external abdominal oblique muscles. This activity coincided with the prolonged expiratory phase that is seen during grunting and which was absent during the inspiratory phase (Fig. 2).

The intubated babies. Recordings from 13 of the 34 babies studied were not used because electrical interference on the traces prevented us from confidently detecting the presence of external abdominal oblique muscle activity. The masking from excessive interference was evident at the same levels

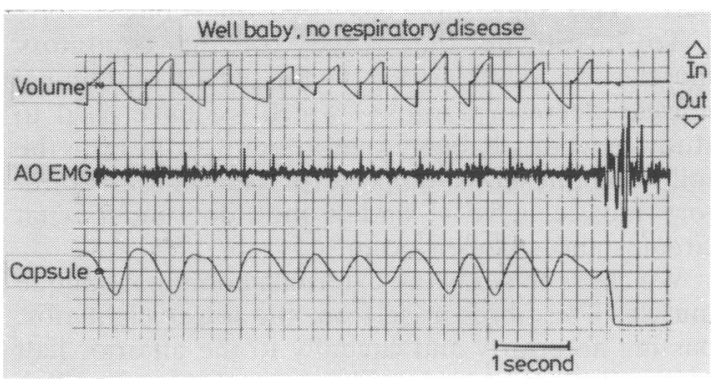

Fig. 1 Respiratory activity in a healthy baby, breathing spontaneously. Volume =tidal volume, from face-mask pneumotachograph; Capsule $=$ signal from pneumatic respiration monitor; $A O E M G=$ abdominal oblique $E M G$ signal; there is no obvious muscle activity in association with respiration, electrocardiogram potentials are visible, and episode to the right of record is a 'wriggle' which shows that the EMG system is working. 


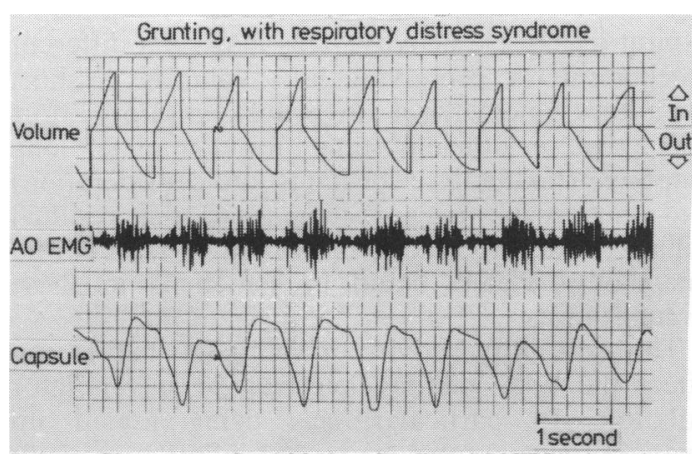

Fig. 2 Respiratory activity in a grunting baby with respiratory distress syndrome. Burst of activity in the abdominal oblique muscles is visible during each expiration.

of signal amplification as the traces which were used. The results were taken from the remaining 21 babies.

During the 30 second period of breathing with CPAP, some external abdominal oblique muscle activity during expiration was observed in all the babies. Although not quantified in any way, the degree of activity in babies on higher CPAP seemed to be greater than those on lower CPAP. In 10 babies we performed a further 30 second observation, beginning with a CPAP of $8 \mathrm{~cm} \mathrm{H}_{2} \mathrm{O}$ and reducing to zero after 15 seconds. In six of these babies the respiratory pattern changed to a faster rate, with either the disappearance of, or a noticeable reduction in, external abdominal oblique muscle activity when lower CPAP was used.

When the babies were receiving IPPV, external abdominal oblique muscle activity was still seen in relation to the baby's spontaneous respiratory cycles, and did not seem to be specifically stimulated by ventilator inflations. If a ventilator inflation coincided with spontaneous expiration there were three possible effects on the tidal volume delivered to the baby by the ventilator. Firstly the baby's expiration had no affect on the tidal volume (Fig. $3 a)$; secondly, the tidal volume was reduced compared with an unaffected inflation (Fig. 3b); and finally, the baby's expiratory efforts reduced the tidal volume delivered and in addition, forced gas up the endotracheal tube against the inflating pressure (Fig. 3c). The first two patterns were often seen in all the babies in the absence of external abdominal oblique muscle activity. The third pattern is of particular interest. We determined how commonly it was observed (Fig. 4).

We analysed 2150 ventilator cycles from the 21 babies. Eighteen babies $(86 \%)$ had episodes during
(A)

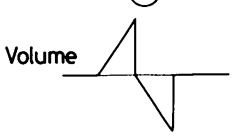

(B)
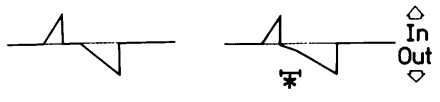

$P$ vent
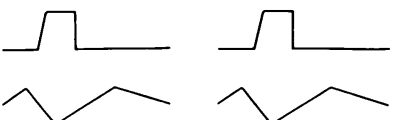

Fig. 3 Diagrammatic respresentation of the effects of expiration by the baby during a ventilator inflation. Top trace: tidal volume (gas flow down endotracheal tube shown as upward deflections and gas flow up tube as downward deflections. Middle trace: ventilator circuit pressure. Bottom trace: respiratory efforts (inspiration downward deflection, expiration upward). A: baby's expiration does not influence tidal volume delivered by ventilator. B: baby's expiration has reduced tidal volume delivered. C: tidal volume is reduced and in addition there is gas flow up endotracheal tube against inflation pressure (during the period marked ${ }^{*}$ ).

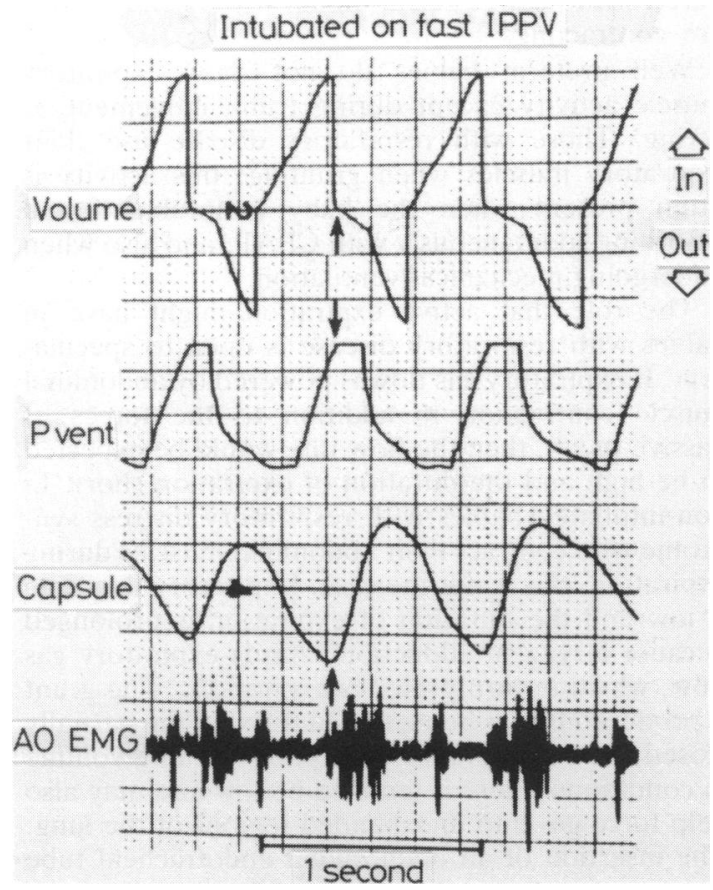

Fig. 4 Respiratory activity in a ventilated baby with respiratory distress syndrome. This shows pattern schematically represented in figure $3 c$. Signals are as shown in figures 1 and 2 , with the addition of ventilator circuit pressure $(P V E N T)$. During each ventilator cycle baby moves into an expiratory phase at a point when ventilator is reaching peak inflation pressure (arrows). Small amount of gas is forced up endotracheal tube against the inflation pressure. Note burst of abdominal oblique activity. 
which part of the ventilator inflation coincided with the baby's expiratory phase and gas flowed up the endotracheal tube against the ventilator inflation pressure. Overall, this pattern was seen during 441 $(21 \%)$ of the 2150 ventilator inflations, and in individual babies its occurrence varied from 0 to $88 \%$ of the analysed breaths. Of these 441 episodes of expiratory flow during ventilator inflation, 432 $(98 \%)$ were associated with a detectable burst of activity in the external abdominal oblique muscles.

\section{Discussion}

We have shown that there is respiratory activity of the external abdominal oblique muscles in preterm babies. This activity coincides with expiration and it seems reasonable to conclude that, as in adults these muscles may provide an active component to expiration. Showing that the expiratory muscles are active by electromyography, however, does not quantify their contribution to expiratory forces when compared with passive recoil alone; it only shows they are contracting.

Well preterm babies do not show expiratory muscle activity except during trunk movement or crying. Those with respiratory disease use their expiratory muscles when grunting; this activity is often present when the baby is intubated and breathing spontaneously with CPAP, and also when undergoing mechanical ventilation.

The role that active expiration might have in babies with respiratory disease is open to speculation. If expiratory gas flow is powered by abdominal muscle contraction in addition to the forces of passive recoil, then the flow rate would be expected to be high and the duration of expiration short. In non-intubated babies with respiratory distress syndrome who contract their abdominal muscles during expiration, this is not the case. Expiratory flow rate is low and the duration of expiration is prolonged because laryngeal adduction retards expiratory gas flow, which in turn prolongs expiration. ${ }^{8}$ The grunt is produced by gas being forced through the partially closed larynx. This may help preserve lung volume in conditions where it tends to be low and may also help force gas into unexpanded regions of the lung. The insertion of an open ended endotracheal tube prevents laryngeal adduction and deprives the baby of this mechanism to maintain lung volume. Deterioration of oxygenation usually ensues. ${ }^{2}$ We have observed that an intubated baby breathing against $8 \mathrm{~cm} \mathrm{H}_{2} \mathrm{O}$ of CPAP behaves like a grunting baby, with a prolonged expiratory time and a lot of expiratory muscle activity. If CPAP is reduced to zero some of these babies switch to a fast respiratory rate with a short expiratory duration and very little expiratory muscle activity. This may be a different mechanism for preserving lung volume. A short expiratory time without any active expiratory effort may give insufficient time for complete lung emptying (taking time constants into consideration) and thus result in a higher functional residual capacity. This dynamic increase in lung volume from 'inapparent PEEP' could be beneficial in these circumstances.

The expiratory activity of ventilated babies can affect the tidal volume delivered by a ventilator inflation. In particular, gas being forced up the endotracheal tube against the inflation pressure is almost consistently associated with abdominal muscle contraction.

Can preterm babies contract their expiratory muscles sufficiently to overcome the ventilator inflation pressure, which in some circumstances may be more than $30 \mathrm{~cm} \mathrm{H}_{2} \mathrm{O}$ ? In the ventilated baby who is also breathing spontaneously the forces expanding the lungs comprise the negative pressure generated during inspiration by the baby and the positive pressure applied by the ventilator. At the peak of any given tidal inflation these forces will be balanced by the elastic recoil of the lungs and chest wall. At this point, either the subtraction of the baby's inspiratory efforts or the addition of an expiratory force-that is, contraction of the expiratory muscles or both-may cause expiratory forces to exceed inspiratory forces which results in gas being forced up the endotracheal tube against peak inflation pressure. Interestingly expiratory muscle activity was almost always seen in these babies. The interaction of these forces is complex and may well vary from breath to breath.

If a force is applied to the chest by the expiratory muscles during ventilator inflation high intrapulmonary pressures may result. Distribution of intrathoracic pressure and lung compliance is not uniform, so gas may be forced into the more compliant compartments (particularly the larger airways), leading to rupture. ${ }^{9}$ This may explain the association of pneumothorax with this particular pattern of baby-ventilator interaction.

Newborn babies with respiratory disease often contract their abdominal muscles during expiration. This can affect the efficacy of treatment using ventilators and add to its complications. The spontaneous respiratory activity of ventilated babies is an important component of ventilation and should not be ignored.

We thank Professor J A Davis. Dr N R C Roberton and Dr G Gandy for their encouragement and advice, and the nursing staff of the neonatal unit at the Rosie Maternity Hospital. This work is funded by the University of Cambridge Baby Research Fund. 


\section{References}

1 Camphell EJM, Green JN. The expiratory function of the abdominal muscles in man. An electromyography study. J Physiol (Lond) 1953:120:409-18.

2 Harrison VC. Heese $H$ de V. Klein M. The significance of grunting in hyaline membrane disease. Pediatrics 1968:41: $549-59$.

3 South M, Morley CJ. Spontanaeous respiratory timing in intubated neonates with R.D.S. Early Hum Dev 1986:14:147-8.

+ Greenough A, Morlcy CJ. Wood S, Davis JA. Pancuronium prevents pneumothoraces in ventilated premature babies who actively expire against positive pressure ventilation. Lancet $1984 ; i: 1-3$.

5 Ramsden A. Active expiration or synchrony? Arch Dis Child 1986:61:820

"South M, Morley CJ, Hughes G. A simple technique for monitoring the electromyogram of the external abdominal oblique muscle in the newborn. Early Hum Dev (In press).

7 South M, Morley CJ. Monitoring spontaneous respiration in the ventilated nconate. Arch Dis Child 1986:61:291-4.

\& Johnson P, Harding R, McClelland M, Whyte P. Laryngeal influence on lung expansion and breathing in lambs. Pediatr Res 1977:11:1025.

${ }^{9}$ LeSoucf PN, Lopes JM, England SJ, Bryan MH, Bryan AC. Influence of chest wall distortion on esophageal pressure. J Appl Physiol 1983;55:353-8.

Correspondence and requests for reprints to $\operatorname{Dr} M$ South, Department of Paediatrics, University of Cambridge, Level E8, New Addenbrooke's Hospital, Cambridge CB2 2QQ.

Received 10 April 1987

\title{
Fifty years ago
}

\section{Iron deficiency anaemia of late infancy}

\author{
HW Fullerton (Aberdeen)_Arch Dis Child 1937;12:91-110
}

This was the first British assessment of the frequency of nutritional anaemia in infancy to follow Dr Helen Mackay's extensive studies in London in 1933. Haemoglobin estimations by the Haldane method were made in 789 infants of all ages up to 23 months. The incidence of severe anaemia increased towards the end of the first year and continued to be high throughout the second year. Haemoglobin concentrations $<80 \%(11.8 \mathrm{~g} / \mathrm{dl})$ were found in $87 \%$ of 298 infants of the "poorer classes" between the ages of 9 and 23 months. Fullerton concluded that the common anaemia of late infancy was due mainly to iron deficiency, which was dependent on low birth weight, prolonged milk (especially artificial) feeding, and infective illnesses. Maternal iron deficiency was regarded as having little or no influence on the development of anaemia in infancy. Analysis of the quantitative aspects of iron deficiency led to the conclusion that a deficient intake of iron during the first nine months did not have a pronounced effect on the haemoglobin concentration but that a deficient iron intake would become of increasing importance as growth continued. Fullerton saw no need for drastic dietary revisions but he did recommend the prescription of medicinal iron from the age of 3 months to all infants of birth weight less than $7 \mathrm{lb}(3200 \mathrm{~g})$ and to all who were artificially fed.

Comment. Iron deficiency anaemia is less common today ( $32 \%$ of Glasgow children between 6 and 24 months). Infants with very severe anaemia and cardiomegaly complicated by infection and requiring emergency blood transfusion were not uncommon before the second world war. They are almost unknown today. In the 1930s mixed feeding was not commonly introduced before the age of 9 months and the present practice of starting mixed feeding, including iron fortified cereals, between the ages of 3 and 6 months probably has a preventive role. A case can still be made, however, for testing infants aged 9 to 12 months, especially in underprivileged families, and prescribing medicinal iron to those found to have anaemia. The need for iron supplements in premature babies is well recognised.

(Harold Fullerton was not a paediatrician but a general physician with a special interest in haematology. He later became Professor of Medicine in the University of Aberdeen.) 